\title{
Estandarización del Instrumento EGMA para la evaluación de habilidades numéricas iniciales en estudiantes de educación básica de Chile*
}

\author{
Standardization of the EGMA Instrument for the evaluation \\ of mathematics in the initial levels of Basic Education
}

\author{
Mariela Elizabeth Cáceres Sandoval ${ }^{a}$ Felipe Sepúlveda ${ }^{a, b}$, Cristina Rodríguez ${ }^{a, c}$ \\ ${ }^{a}$ Facultad de Educación, Universidad Católica de la Santísima Concepción, Concepción, Chile. \\ mcaceres@magister.ucsc.cl, fsepulveda@ucsc.cl \\ ${ }^{b}$ Centro de Investigación en Educación y Desarrollo, CIEDE-UCSC. \\ ${ }^{c}$ Facultad de Psicología, Universidad de La Laguna, Tenerife, España. \\ crodri@ull.edu.es
}

\section{RESUMEN}

La evaluación de habilidades numéricas temprana es fundamental de cara a la intervención. En este sentido, es necesario contar con un instrumento fiable que permita la detección para crear planes de apoyo y dar respuesta a las necesidades específicas de los estudiantes. El presente trabajo tiene por objetivo estandarizar el instrumento de evaluación EGMA, para estudiantes de primer a tercer año básico. Esta herramienta mide conocimientos matemáticos tempranos esenciales y predictivos de logros posteriores utilizando tareas relacionadas con identificación y discriminación numérica, número faltante, adición, sustracción y problemas de palabras. Se analizó una muestra de 289 estudiantes de la región del Biobío, Chile. El instrumento de evaluación EGMA presentó adecuados indicadores de consistencia interna. Se reportan los estadísticos descriptivos y baremos para la muestra analizada.
\end{abstract}

Palabras claves: habilidades numéricas, matemáticas, intervención temprana, evaluación.

\section{ABSTRACT}

The assessment of the early mathematics skills is the key for the intervention. In this regard, it is needed to count with a reliable instrument which allows the detection to create the supported plan required and give an answer to the specific needs of the students. The objective of this work is to standardize the assessment instrument EGMA, for students from the 1st grade to 3rd grade. This tool assess early mathematical essential knowledge and gives a prediction about later outcomes through the use of tasks related to identification and numerical discrimination, missing number, addition, subtraction and word problems. A sample of 289 students was analyzed in the Bio- Bio region, Chile. This assessment instrument EGMA show the appropriate indicators of intern consistency. It was reported descriptive statistics and the scales of the analyzed sample.

Key words: numerical skills, mathematics, early intervention, evaluation.

\footnotetext{
* La investigación fue parcialmente financiada por el proyecto CONICYT, FONDECYT REGULAR 1161213 del Ministerio de Educación de Chile y el Ministerio de Economía y Competitividad de España, Programa Ramón y Cajal, RYC-201416948.
} 
Estudios Pedagógicos XLVI N 1: 301-318, 2020

ESTANDARIZACIÓN DEL INSTRUMENTO EGMA PARA LA EVALUACIÓN DE HABILIDADES NUMÉRICAS INICIALES EN ESTUDIANTES DE EDUCACIÓN BÁSICA DE CHILE

\section{INTRODUCCIÓN}

En los últimos 20 años, las políticas educacionales implementadas en Chile se han centrado en desarrollar infraestructura de establecimientos educativos, ampliar el acceso y fortalecer la formación inicial docente. Al estar estas necesidades adecuadamente cubiertas, últimamente el énfasis en torno a la reforma educacional ha apuntado en mejorar la calidad de la enseñanza. De este modo, el Ministerio de Educación Chileno reconoce que es preciso que las distintas políticas educativas se dirijan a optimizar el aprendizaje de todos los estudiantes, poniendo especial énfasis en aquellos que presentan bajos desempeños o que de alguna u otra forma van quedando rezagados (MINEDUC, 2016).

La matemática se muestra como una de las asignaturas fundamentales dentro del currículum nacional ya que su aprendizaje permite comprender la realidad y proporciona herramientas necesarias para desenvolverse en la vida cotidiana, contribuyendo así al pensamiento lógico, ordenado, crítico y autónomo y de actitudes como la rigurosidad, la perseverancia y la confianza en sí mismo (MINEDUC, 2012). Es por tanto esencial que los niños y niñas adquieran los objetivos de aprendizaje que se proponen para esta asignatura y puedan ir avanzando en el logro de estos. En las distintas evaluaciones, tanto internacionales como nacionales, los puntajes obtenidos por el alumnado chileno no muestran avances significativos en los últimos años.

La Agencia de Calidad de la Educación Chilena (ACE) a través de Sistema de Medición de la Calidad de la Educación (SIMCE) evalúa los resultados de aprendizaje de los establecimientos, midiendo el logro de los contenidos y habilidades del currículo vigente, en diferentes asignaturas o áreas de aprendizaje. Inicialmente el SIMCE evaluaba en lectura y matemáticas a todos los estudiantes empezando en el segundo curso de educación básica. Sin embargo, a partir del año 2016 el SIMCE se comienza a aplicar desde cuarto básico dejando un vacío de información que pudiese ser relevante para la detección temprana de trayectorias de aprendizaje disminuidas. Los resultados a nivel nacional constatan que desde el año 2011 los estudiantes que cursan cuarto básico han mantenido su desempeño en matemática donde el $75.6 \%$ de los estudiantes evaluados se desempeñan en las categorías de elemental o insuficiente con respecto a los estándares de aprendizaje en este nivel (Agencia de Calidad de la Educación, 2017).

En la actualidad, los distintos instrumentos que se aplican tanto a nivel nacional como internacional no apuntan a los primeros niveles de educación, por lo que se hace necesario aplicar evaluaciones que entreguen información valiosa acerca de lo que sucede en los primeros años de la enseñanza formal. Para esto, las pruebas de screening validadas y de libre acceso son útiles para que todos los profesores puedan tener un panorama amplio de los aprendizajes que los niños y niñas van alcanzando. Este tipo de instrumentos de evaluación deben contar con la sensibilidad suficiente para determinar qué habilidades matemáticas específicas a este nivel se encuentran descendidas, y así poder establecer metas de aprendizaje coherentes con las necesidades individuales de cada estudiante. Con este fin, en el último tiempo se han diseñado las pruebas CBM (por sus siglas en inglés, Curriculum Based Measures) dirigidas a evaluar el dominio de objetivos de aprendizaje a ser enseñados en un determinado período escolar. El propósito esencial de las pruebas CBM es permitir a los docentes evaluar la efectividad de su instrucción a cada uno de sus estudiantes (Deno, 2003). Este tipo de evaluaciones son muy utilizadas en el sistema escolar norteamericano, sin embargo, las pruebas de habilidades matemáticas CBM validadas para estudiantes de 
países hispanos son muy limitadas. Una excepción es el reciente trabajo de León et al. (2020) dirigido a la validación de la prueba de Indicadores de Progreso de Aprendizaje en Matemáticas en estudiantes de primer año básico de Tenerife, España.

Esta investigación tiene como principal objetivo estandarizar el instrumento EGMA (Early Grades Mathematics Assesment), herramienta CBM diseñada por Research Triangle Institute (RTI) como una prueba de screening de libre acceso útil para estimar el desarrollo de competencias matemáticas iniciales que son predictoras de futuros aprendizajes matemáticos de mayor complejidad. Se informan indicadores de la estructura interna del instrumento EGMA basado en una muestra de estudiantes de primer a tercer año básico en Chile.

\section{MARCO TEÓRICO}

\subsection{HABILIDADES NUMÉRICAS TEMPRANAS}

La instrucción de habilidades numéricas se encuentra presente en el currículo desde las primeras etapas de la trayectoria escolar. En esta etapa inicial, el desarrollo de competencias numéricas claves son la base para la posterior adquisición de conceptos matemáticos más complejos y pueden ser predictores del posterior éxito académico de los estudiantes (Andrews \& Sayers, 2013; Aunio \& Niemivirta, 2010). En los últimos 20 años, se han registrado importantes avances en la investigación relativa a la identificación de habilidades numéricas iniciales relevantes de ser incorporadas a la instrucción formal. Así, existe un relativo acuerdo entre los investigadores del área con respecto a los indicadores claves que representan el nivel de desarrollo de las habilidades numéricas iniciales tales como: el conteo oral, la discriminación de cantidades, la identificación de cantidades, la identificación de números y la identificación de un número faltante dada secuencia numérica (Clarke \& Shinn, 2004; Jordan, Glutting, Ramineni \& Watkins, 2010; Purpura \& Lonigan, 2015). El apropiado desarrollo de estas habilidades numéricas facilita el aprendizaje de conceptos matemáticos más complejos asociados con el cálculo y la resolución de problemas.

El conteo se ha identificado como una de las habilidades numéricas tempranas relevantes para el desarrollo de las matemáticas. Los cinco principios del conteo propuestos por Gelman \& Gallister (1978) y su posterior trayectoria de aprendizaje desarrollada por Clements \& Sarama, (2009), todavía son reconocidas como bases fundamentales en la enseñanza en las primeras etapas de la formación escolar (Jacobi-Vessels, Todd Brown, Molfese \& Do, 2016). El dominio efectivo de las diferentes fases que componen la habilidad del conteo (tales como la correspondencia uno a uno, cardinalidad y la irrelevancia del orden) son importantes para la resolución de problemas matemáticos simples que requieren algún tipo de comprensión aritmética, (Geary, 2011).

Al igual que el conteo, la identificación de los números ha sido descrita como una destreza relevante para el desarrollo del pensamiento matemático. Un estudio longitudinal que trabajo con estudiantes desde los 6 años de edad encontró que la identificación numérica era un consistente predictor de crecimiento en las habilidades aritméticas de los participantes (Göbel, Watson, Lervåg \& Hulme, 2014). Adicionalmente a la identificación numérica, diversos estudios se han centrado en la importancia en la comparación simbólica de cantidades como un nexo importante en las estrategias para resolver problemas matemáticos de manera efectiva y eficiente (Clarke, Baker, Smolkowski \& Chard, 2008). Así, otro estudio 
Estudios Pedagógicos XLVI N 1: 301-318, 2020

ESTANDARIZACIÓN DEL INSTRUMENTO EGMA PARA LA EVALUACIÓN DE HABILIDADES NUMÉRICAS INICIALES EN ESTUDIANTES DE EDUCACIÓN BÁSICA DE CHILE

longitudinal encontró una alta correlación entre el procesamiento numérico simbólico y dos mediciones independientes de habilidades aritméticas (Vanbinst, Ansari, Ghesquière \& Smedt, 2016).

\subsection{RESULTADOS CHILENOS NACIONALES E INTERNACIONALES EN LA ASIGNATURA DE MATEMÁTICA}

En relación con los logros académicos que alcanzan los estudiantes chilenos en la asignatura de matemática, estos son evaluados a través de distintos instrumentos, tanto nacionales como internacionales. A nivel internacional, Chile participa principalmente de tres estudios, que son el Programa para la Evaluación internacional de Estudiantes (PISA) elaborado por la Organización para la Cooperación y el Desarrollo Económico (OCDE), el cual busca evaluar el nivel de competencias que poseen estudiantes para una completa participación en la sociedad cuando estos están próximos a terminar su etapa escolar; el Estudio Regional Comparativo y Explicativo (ERCE), desarrollado por el laboratorio Latinoamericano de Evaluación de la Calidad de Educación que entrega información sobre los logros de aprendizaje de los países de América Latina y el Caribe y el Estudio Internacional de Tendencias en Matemática y Ciencias (TIMMS) llevado a cabo por la Asociación Internacional para la Evaluación del Logro Educativo (IEA). Según se indica en la Agencia de la Calidad de la Educación Chilena (2018):

La participación de Chile en estos estudios permite contar con información relevante para monitorear el sistema educativo, el currículo vigente, las políticas públicas en educación y los programas que se han implementado, además de incorporar estándares internacionales a las evaluaciones y marcos de estudios nacionales (p. 18).

Con respecto al informe PISA, Chile se incorpora por primera vez en la evaluación en profundidad de Matemática en el año 2012 ya que no participó en el estudio anterior del 2003 que había considerado como área principal esta asignatura. En este sentido, es posible establecer que los puntajes obtenidos por los estudiantes chilenos en Matemática se han mantenido estables durante las últimas tres aplicaciones, mostrando que sus competencias se han mantenido sin variación estadísticamente significativa, sin evidenciarse de este modo un retroceso en el desarrollo de las competencias por parte de los estudiantes chilenos. Chile, al igual que los países latinoamericanos que participan en el estudio PISA, se mantiene igual, sin alzas ni bajas en los puntajes.

En el Segundo y Tercer Estudio Regional Comparativo y Explicativo (SERCE y TERCE), la evaluación matemática se ha planteado en base a la revisión del Currículo nacional de cada uno de los países participantes, considerando dominios y procesos cognitivos (UNESCO, 2016). Este estudio evalúa el desempeño escolar en tercero y sexto año básico en las áreas de matemática, lenguaje y desde la tercera aplicación Ciencias Naturales en sexto básico. Los resultados promedio de los alumnos de $3^{\circ}$ y $6^{\circ}$ básico en las aplicaciones de SERCE y TERCE en matemática sitúan a Chile en el grupo de países con promedios significativamente sobre la media internacional (Agencia de Calidad de la Educación, 2018). En tercero básico entre los años 2006 y 2013, existe un aumento significativo de 529 a 582 puntos. Lo mismo sucede en el sexto año básico donde existe un aumento de 63 puntos en este mismo periodo. El Informe TERCE del año 2015 muestra, 
además, que existe una clara tendencia en relación con el nivel socioeconómico, donde a mayor quintil de los estudiantes mayores fueron los resultados en el área de matemática, tanto de tercero como de sexto año básico. Sin embargo, en este último curso las diferencias fueron más altas, lo que podría indicar que las diferencias socioeconómicas se van enfatizando a medida que avanza el tiempo (Agencia de Calidad de la Educación, 2015).

El estudio TIMSS se aplica desde 1995 en ciclos de cuatro años a estudiantes de $4^{\circ}$ y $8^{\circ}$ básico en las áreas de Ciencia y Matemática. Chile participa en TIMSS desde 1999 evaluando a estudiantes de $8^{\circ}$ básico y desde 2011 evaluando a estudiantes de $4^{\circ}$ básico. La escala de puntaje tiene un rango de 0 a 1000 puntos, centrada en 500. En el periodo del 2011 al 2015, en el área de matemática, en los cursos de cuarto y octavo año básico se encuentran bajo el centro de la escala, pero con una tendencia al alza en los resultados en octavo año básico. En cuarto básico los resultados se han mantenido estables, con una leve disminución de 3 puntos, pero que no es significativa. En octavo básico se evidencia un alza significativa de los resultados en comparación a la aplicación de 1999, con una tendencia al alza desde la aplicación del 2003 (Agencia de Calidad de la Educación, 2015).

Con respecto a las pruebas nacionales, se puede establecer que el SIMCE es la única evaluación que se aplica a los estudiantes para conocer los resultados de aprendizajes que están adquiriendo durante el proceso educativo. Según el reporte de calidad del año 2012, elaborado por la Agencia de Calidad, desde el año 1998 se aplica el Simce. Los resultados de esta prueba entregan información de los Estándares de Aprendizaje logrados por los estudiantes en los distintos niveles de enseñanza, complementando el análisis que realiza cada establecimiento a partir de sus evaluaciones y de esta forma situar los logros a nivel nacional. Los Estándares de Aprendizaje son referentes que describen lo que los estudiantes deben saber y poder hacer, para demostrar determinados niveles de cumplimiento de los Objetivos de Aprendizaje estipulados en el currículum vigente (MINEDUC, 2014).

En los resultados del Simce de los últimos 10 años en la asignatura de matemática de cuarto año básico se observa estabilidad desde el año 2011 además de reducirse las diferencias entre los grupos socioeconómicos. El puntaje obtenido en el 2017 es de 261 puntos, uno más que el año anterior. En relación con sexto año básico los resultados se han mantenido relativamente estables con respecto a la aplicación del año 2013 (Agencia de Calidad de la Educación, 2018).

\subsection{PRUEBAS DE MEDICIÓN HABILIDADES MATEMÁTICAS TEMPRANAS}

La evaluación de matemática a nivel inicial se lleva a cabo a través de algunas pruebas aplicadas en los centros educativos, iniciando en educación Parvularia y los primeros años de la Educación General Básica, siendo estos instrumentos esenciales para obtener información relevante e intentar obtener mejoras en los procesos educativos de los niños y niñas, sin embargo, estas Pruebas no son evaluaciones a las que todos los colegios y escuelas tienen acceso, debido principalmente, al costo que estas demandan. Las principales evaluaciones que se pueden encontrar se detallan a continuación.

\subsubsection{Prueba EGMA (Early Grades Mathematic Assessment)}

La preocupación por la calidad educativa ha llevado a varios organismos internacionales, entre ellos el Banco Mundial (BM) y el Research Triangle Institute (RTI), a impulsar 
medidas encaminadas a mejorar el aprendizaje en países como Nicaragua a traves del Centro de Investigación y Acción Educativa Social (CIASES) . Entre estas medidas se encuentra el uso de la evaluación como un mecanismo tanto correctivo como preventivo (CIASES y RTI Internacional, 2011). El RTI, ha desarrollado metodologías para la medición de habilidades lectoras y destrezas matemáticas en los primeros grados de primaria. Para esto el RTI ha construido pruebas cortas y orales, la prueba utilizada para la evaluación de habilidades matemáticas es conocida como EGMA (Early Grades Mathematics Assessment).

La prueba EGMA mide conocimientos matemáticos tempranos y habilidades que son fundamentales y predictivas de logros posteriores, incluyendo en su evaluación la identificación del número, la discriminación numérica, los patrones numéricos y la suma y resta. La administración de esta prueba puede proporcionar a los encargados de formular políticas, investigadores, información relevante sobre si las políticas educativas existentes, programas curriculares y las intervenciones en el aprendizaje están apoyando a los estudiantes a alcanzar las metas en matemática (Platas, Ketterlin-Geller y Sitabkhan, 2016).

Según el Informe de resultados EGMA de Nicaragua (2011) el RTI tomó en cuenta dos enfoques para la elaboración de la prueba EGMA. El primero consistió en revisar el currículo y objetivos de varios estados y países para kínder, primero y segundo grado y el segundo buscaba identificar medidas que fueran representativas de cada grado, para asegurar que se considerara el progreso en habilidades que permitían el desarrollo de competencias matemáticas. El segundo implicó la revisión los objetivos establecidos por la NCTM por sus siglas en inglés (National Council of Teachers of Mathematics), los hallazgos reportados por el National Mathematics Advisory Panel y finalmente la influencia de TIMSS en cada uno de ellos. La revisión de los currículos de diferentes países permitió a RTI concluir que los principales contenidos para los primeros grados son muy similares, lo que facilitó la preparación de EGMA, de acuerdo con estos puntos de referencia los estudiantes deberían demostrar cierto nivel de comprensión de los números enteros (identificar, ordenar, sumar y restar); demostrar comprensión de patrones, como patrones de extensión que utilizan secuencias numéricas y/o geométricas; ser capaz de identificar el siguiente elemento de la secuencia o el elemento que completa una secuencia y resolver problemas de contexto cotidiano (CIASES y RTI Internacional, 2011).

\subsubsection{Prueba de evaluación matemática temprana Utrecht (TEMT-U)}

El TEMT evalúa el nivel de competencia matemática temprana en niños de 4 a 7 años, requisito para poder seguir en los años siguientes una educación matemática formal. El TEMT-U corresponde a la versión española del Utrecht Early Numeracy Test, creado por Johannes van Luit, Bernadette van Rijt y Albert Pennings, en 1994 examina dos dimensiones de la competencia matemática temprana: subtest relacionales y numéricos. Dispone de tres formas paralelas (versiones A, B y C), de 40 ítems cada una. Consta de 8 áreas de competencia, en grupos de 5 ítems cada una. El Test tiene una puntuación máxima de 40 puntos, uno por cada ítem correcto. Las dimensiones que incluye son conceptos de comparación, clasificación, correspondencia uno a uno, seriación, conteo verbal (uso de secuencia numérica oral), conteo estructurado y conteo resultante o resultado del conteo (Cerda et al., 2012).

Esta prueba presenta ciertas ventajas como, facilitar mediante un screening rápido el conocimiento matemático de los niños y niñas, además de entregar información sobre 
los procesos lógicos y numéricos involucrados en el conocimiento matemático temprano (Navarro, Aguilar, Marchena, Alcalde y García, 2010).

Con respecto a esta evaluación, se realiza un estudio en Chile, relacionado con la validez y confiabilidad de la prueba, en el año 2012, el que concluye que este instrumento puede ser considerado útil para evaluar el desempeño en el área de matemática de estudiantes de Segundo ciclo de Educación de Párvulos y primer ciclo básico, en la medida que se reportan puntajes comparativos por edad, nivel educativo y dependencia administrativa (Cerda et al., 2012).

Con relación a esta misma prueba, se realiza un estudio en España, administrando este instrumento a 127 alumnos de $3^{\circ}$ de educación infantil, en tres colegios; cuyo objetivo fue conocer el desarrollo matemático de los estudiantes en sus aspectos relacionales y de conteo. Con respecto a los principales resultados se puede establecer que en los subtest de comparación y clasificación la muestra obtiene buenos resultados, también que los subtest de Conteo y Seriación son los más difíciles para los niños de este grupo (Navarro et al., 2010).

\subsubsection{Prueba TEMA-3 (Test of Early Mathematic Ability $3^{\text {rd }}$ Edition)}

Esta prueba de los autores Ginsburg y Baroody del año 2007, es una prueba de aplicación individual en un tiempo variable, entre 30 y 45 minutos destinado a niños y niñas entre 3 a 8 años 11 meses de edad. Permite identificar desde las primeras etapas de escolaridad alumnos con dificultades de aprendizaje o que pudieran llegar a desarrollarlas. Es un test normativo, fiable y válido de la habilidad matemática infantil. No es un test cronometrado, por esto no se impone límite de tiempo. Está compuesto por 72 ítems que valoran diferentes aspectos de la competencia matemática básica. Los aspectos informales de las matemáticas, es decir aquellas habilidades que no precisan el uso de símbolos escritos, son valorados en 41 ítems, que se reparten en cuatro categorías relacionadas con la numeración, comparación de cantidades, habilidades del cálculo informal y conceptos.

La escala formal, relacionada con actividades que implican el uso de los símbolos matemáticos, está compuesta por 31 ítems, distribuidos en 4 componentes relacionados con el conocimiento de convencionalismos, hechos numéricos, habilidades de cálculo y componentes de base 10 .

Los resultados de las pruebas son simples observaciones, no diagnósticos. Especifican el nivel de ejecución en un momento dado, en una situación concreta, pero no brindan información de por qué el niño o niña evaluada resuelve de esa manera.

Este test tiene como principal propósito proporcionar información útil sobre el nivel de competencia matemática de los niños más pequeños. Los resultados obtenidos pueden tener distintas utilidades, como por ejemplo identificar a niños y niñas que manifiesten un nivel de desarrollo matemático significativamente mejor o peor que sus iguales, identificar las fortalezas y debilidades específicas en la competencia matemática de niños y niñas, orientar las prácticas educativas apropiadas para tratamientos individuales, registrar el progreso en el aprendizaje aritmético de los alumnos o la eficacia de los programas de intervención.

En la tabla 1, se puede observar un resumen sobre las pruebas descritas anteriormente y las principales características en cuanto a las habilidades matemáticas que evalúan, edad de aplicación y disponibilidad en Chile. 
Estudios Pedagógicos XLVI N 1: 301-318, 2020

ESTANDARIZACIÓN DEL INSTRUMENTO EGMA PARA LA EVALUACIÓN DE HABILIDADES NUMÉRICAS INICIALES EN ESTUDIANTES DE EDUCACIÓN BÁSICA DE CHILE

Tabla 1. Pruebas para evaluar habilidades matemáticas temprana

\begin{tabular}{|l|l|l|l|}
\hline Prueba & Habilidades matemáticas evaluadas & Edad-nivel & Disponibilidad en Chile \\
\hline EGMA & $\begin{array}{l}\text { Identificación de números } \\
\text { Discriminación numérica } \\
\text { Secuencias o patrones numéricos } \\
\text { Adición, - sustracción } \\
\text { Resolución de problemas }\end{array}$ & $\begin{array}{l}\text { Primero a cuarto año } \\
\text { básico }\end{array}$ & Gratuita \\
\hline TEMT-U & $\begin{array}{l}\text { Concepto de comparación } \\
\text { Clasificación } \\
\text { Comparación uno a uno } \\
\text { Seriación } \\
\text { Conteo verbal } \\
\text { Conteo estructurado } \\
\text { Conteo resultante } \\
\text { Conocimiento general de los } \\
\text { números }\end{array}$ & 4 -7 años de edad & Valor comercial \\
\hline TEMA-3 & $\begin{array}{l}\text { Numeración } \\
\text { Comparación de cantidades } \\
\text { Habilidades del cálculo mental } \\
\text { Conceptos }\end{array}$ & $\begin{array}{l}3 \text { a } 8 \text { años 11 meses } \\
\text { de edad }\end{array}$ & Valor comercial \\
\hline US\$ 169 (set completo
\end{tabular}

Fuente. Elaboración propia.

\section{METODOLOGÍA}

La presente investigación es de tipo no experimental, descriptiva y transeccional, tiene como principal objetivo validar un instrumento screening de matemática (EGMA) para los niveles iniciales de educación básica.

\subsection{PROCEDIMIENTO}

Se administró la prueba EGMA en forma individual a los niveles de primero, segundo y tercer año básico de tres Colegios de la Comuna de Concepción, Hualpén, Talcahuano y San Pedro de la Paz. Responder al test lleva aproximadamente 15 a 20 minutos, considerando el curso en el que se encuentran los niños y niñas. Todos los ítems fueron presentados oralmente y los niños y niñas respondieron señalando en forma verbal y escrita, utilizando en algunos casos, estrategias como uso de dedos, lápiz y papel o contestando con cálculo mental.

Antes del inicio de la administración de las pruebas, se realizó una entrevista con cada uno de los directores de los establecimientos para exponer objetivos del proyecto. A partir de esto, los profesores fueron informados acerca de la aplicación de la prueba, solicitando, además, la autorización a las familias para que los participantes fueran evaluados con el 
instrumento presentado. Se procedió a realizar las evaluaciones, donde cada estudiante consintió en forma verbal el participar de este proceso.

El análisis de datos fue desarrollado con la herramienta ULLRtoolbox (Hernández, 2019) que opera bajo el ambiente de R y las gráficas fueron diseñadas utilizando SPSS, v.23.

\subsection{PARTICIPANTES}

Los participantes de esta investigación están constituidos por 5 establecimientos ubicados en las ciudades de Concepción, Hualpén, Talcahuano y San Pedro de la Paz. La muestra determinada para esta investigación está compuesta por 289 estudiantes, como lo muestra la tabla 2, pertenecientes a primer, segundo y tercer año básico de los colegios antes mencionados. La tabla 3 presenta la muestra distribuida según sexo, considerando para este estudio a 137 mujeres y 152 hombres. Esta muestra fue definida por un muestreo no probabilístico, por conveniencia y conglomerados considerando a los estudiantes disponibles para participan en cada centro escolar y los tres niveles considerados en el estudio.

Tabla 2. Distribución de la muestra de estudiantes en función de la Dependencia administrativa y nivel académico

\begin{tabular}{|c|c|c|c|c|c|}
\hline \multirow[b]{2}{*}{ Nivel Educativo } & \multicolumn{3}{|c|}{ Dependencia administrativa } & \multirow[b]{2}{*}{ Total } & \multirow[b]{2}{*}{ Porcentaje } \\
\hline & Municipal & $\begin{array}{c}\text { Particular } \\
\text { Subvencionado }\end{array}$ & Particular & & \\
\hline Primer año básico & 23 & 59 & 29 & 111 & $38,4 \%$ \\
\hline Segundo año básico & & 61 & 30 & 91 & $31,4 \%$ \\
\hline Tercer año básico & & 60 & 27 & 87 & $30.1 \%$ \\
\hline Total & 23 & 180 & 86 & 289 & $100 \%$ \\
\hline
\end{tabular}

Fuente. Elaboración propia.

Tabla 3. Distribución de la muestra de estudiantes según sexo

\begin{tabular}{|c|c|c|c|}
\hline \multirow{2}{*}{ Nivel Educativo } & \multicolumn{2}{|c|}{ Sexo } & \multirow{2}{*}{ Total } \\
\cline { 2 - 4 } & Femenino & 59 & 111 \\
\hline Primer año básico & 52 & 44 & 91 \\
\hline Segundo año básico & 47 & 49 & 87 \\
\hline Tercer año básico & 38 & $\mathbf{1 5 2}$ & $\mathbf{2 8 9}$ \\
\hline Total & $\mathbf{1 3 7}$ & & \multirow{2}{*}{} \\
\hline
\end{tabular}

Fuente. Elaboración propia. 


\subsection{INSTRUMENTO}

El instrumento EGMA mide conocimientos matemáticos tempranos esenciales y habilidades que son fundamentales, predictivas de logros posteriores. Este consta de 6 secciones. Cada ítem acertado se puntúa con 1 y las respuestas incorrectas o las que no se realizan con 0 . La puntuación máxima es de 96 puntos. Las subpruebas se relacionan con:

a) Identificación de números: Mide la fluidez de asociación de un símbolo (o numeral) con su correspondiente nombre. El tiempo máximo para realizar esta subprueba es de 60 segundos. La puntuación esperada es de 20 puntos.

b) Discriminación numérica: Juzga las diferencias cuantitativas que dos números representan. Compara y ordena números naturales. La puntuación máxima es de 10 puntos.

c) Número faltante: Está relacionada con reconocer, completar y extender secuencias numéricas. La puntuación máxima es de 10 puntos.

d) Adición: Evalúa la fluidez del cómputo de sumas. Este ítem cuenta con dos subpruebas; adición nivel 1 y adición nivel 2. En la adición de primer nivel la puntuación máxima es de 20 puntos y la de segundo nivel de 5 puntos.

e) Sustracción: Mide la fluidez del cómputo de restas. Este ítem, al igual que la adición, cuenta con dos subpruebas; sustracción nivel 1 y sustracción nivel 2. En la sustracción de primer nivel la puntuación máxima es de 20 puntos y la de segundo nivel es de 5 puntos.

f) Resolución de problemas: Comprende la aplicación de distintas estrategias para la resolución de problemas. La puntuación máxima es de 6 puntos.

\section{RESULTADOS}

\subsection{CONSISTENCIA INTERNA PARA LA MUESTRA DEL ESTUDIO}

Se calculó la fiabilidad de la prueba EGMA considerando a todos los participantes del estudio. Así, el valor de a de Cronbach para los 96 ítems de la prueba fue de 0.85 representando una alto consistencia interna del instrumento. Luego, de acuerdo a lo recomendado por Field (2013), se procedió a calcular los valores de a de Cronbach para cada sub-tarea incluida en el instrumento. Los resultados de fiabilidad para cada sub-tarea sustentan la alta consistencia interna de instrumento, variando desde 0.48 a 0.86 como se evidencia en la tabla 4 que muestra los resultados obtenidos para el Instrumento EGMA. Los valores más bajos de a se observan en las tareas de adiciones nivel 2 y problemas de palabras que incluyen 5 y 6 ítems en la tarea. Adicionalmente estas tareas no logran ser respondidas por la gran mayoría de los niños en niveles inferiores (primero y segundo) lo que puede estar influyendo en los valores de a reportados.

Tabla 4. Consistencia interna para cada sub-prueba del instrumento EGMA

\begin{tabular}{|l|c|c|}
\hline \multicolumn{1}{|c|}{ Tarea } & Número ítems & Alpha de Cronbach \\
\hline Identificación Numérica & 20 & 0.86 \\
\hline Discriminación Numérica & 10 & 0.76 \\
\hline
\end{tabular}




\begin{tabular}{|l|c|c|}
\hline Numero Faltante & 10 & 0.71 \\
\hline Adiciones Nivel 1 & 20 & 0.80 \\
\hline Adiciones Nivel 2 & 5 & 0.53 \\
\hline Sustracciones Nivel 1 & 20 & 0.79 \\
\hline Sustracciones Nivel 2 & 5 & 0.81 \\
\hline Problema de Palabras & 6 & 0.48 \\
\hline
\end{tabular}

Fuente. Elaboración propia.

En relación con las correlaciones entre las distintas tareas que presenta el instrumento EGMA, como lo muestra la tabla 5, los resultados encontrados en este caso fueron correlaciones altas y significativas entre las diferentes subpruebas.

Tabla 5. Correlaciones entre las tareas del instrumento EGMA

\begin{tabular}{|l|c|c|c|c|c|c|c|c|c|}
\hline & 1 & 2 & 3 & 4 & 5 & 6 & 7 & 8 & 9 \\
\hline 1. Identificación de números & 1 & $.659^{* *}$ & $.694^{* *}$ & $.491^{* *}$ & $.377^{* *}$ & $.473^{* *}$ & $.379^{* *}$ & $.418^{* *}$ & $.783^{* *}$ \\
\hline 2. Discriminación numérica & & 1 & $.533^{* *}$ & $.416^{* *}$ & $.299^{* *}$ & $.449^{* *}$ & $.278^{* *}$ & $.385^{* *}$ & $.665^{* *}$ \\
\hline 3. Número faltante & & & 1 & $.598^{* *}$ & $.494^{* *}$ & $.585^{* *}$ & $.526^{* *}$ & $.555^{* *}$ & $.835^{* *}$ \\
\hline 4. Adición primer nivel & & & & 1 & $.525^{* *}$ & $.721^{* *}$ & $.550^{* *}$ & $.496^{* *}$ & $.833^{* *}$ \\
\hline 5. Adición segundo nivel & & & & & 1 & $.545^{* *}$ & $.543^{* *}$ & $.369^{* *}$ & $.661^{* *}$ \\
\hline 6. Sustracción primer nivel & & & & & & 1 & $.566^{* *}$ & $.544^{* *}$ & $.832^{* *}$ \\
\hline 7. Sustracción segundo nivel & & & & & & & 1 & $.413^{* *}$ & $.667^{* *}$ \\
\hline 8. Problemas de palabras & & & & & & & & 1 & $.662^{* *}$ \\
\hline 9. Puntaje total prueba & & & & & & & & & 1 \\
\hline
\end{tabular}

Fuente. Elaboración propia.

**. La correlación es significativa al nivel 0.01 (bilateral)

\subsection{ANÁLISIS FACTORIAL EXPLORATORIO DE VERSIÓN EGMA ESPAÑOL}

Se llevó a cabo un análisis factorial exploratorio para determinar la estructura interna de EGMA. Se consideró el coeficiente de Kaiser-Meyer-Olkin (KMO) para verificar la utilización de un tamaño de muestra adecuado para el análisis. Así, el resultado de KMO fue de 0.88 y varió entre 0.82 a 0.93 para cada una de las sub-tareas de EGMA, lo que se representa un excelente rango para este análisis (Field, 2013). El test de Bartlett para evaluar la esfericidad de los datos fue significativo $\left(\mathrm{c}^{2}(28)=4023.1, \mathrm{p}<.001\right)$. El análisis factorial exploratorio se ejecutó utilizando un análisis de ejes principales con rotación varimax. Siguiendo el criterio de Kaiser que considera definir el número de factores de 
acuerdo a los eigenvalue sobre 1 , se determinó la existencia de un sólo factor que agrupa las 8 sub-tareas que componen la prueba EGMA que en su conjunto explican el 89\% de la varianza. Las cargas factoriales junto con la comunalidad para cada sub-tarea de EGMA se muestra en la tabla 6.

Tabla 6. Carga factorial subpruebas Instrumento EGMA

\begin{tabular}{|l|c|c|}
\hline \multicolumn{1}{|c|}{ Tarea } & Carga & Comunalidad \\
\hline Identificación Numérica & .700 & .490 \\
\hline Discriminación Numérica & .603 & .364 \\
\hline Numero Faltante & .809 & .655 \\
\hline Adiciones Nivel 1 & .789 & .623 \\
\hline Adiciones Nivel 2 & .636 & .404 \\
\hline Sustracciones Nivel 1 & .800 & .639 \\
\hline Sustracciones Nivel 2 & .661 & .437 \\
\hline Problema de Palabras & .645 & .416 \\
\hline
\end{tabular}

Fuente. Elaboración propia.

\subsection{ANÁLISIS DESCRIPTIVO SUB-PRUEBAS EGMA}

En la figura 1 se presentan los histogramas con el total del puntaje en la prueba EGMA, el desempeño de los niños y niñas fue variando según el curso en el que se aplicó el instrumento. De esta forma, en la medida se progresa en el currículum, los estudiantes registran un aumento progresivo en la puntuación total de EGMA según se observa en el promedio registrado con la línea punteada vertical.

Como se evidencia en la tabla VII para el total de la prueba, los niños y niñas del primer año básico obtuvieron una $\mathrm{M}=42.00$ y $\mathrm{SD}=15.38$; el grupo de segundo año básico obtuvieron una media de $\mathrm{M}=53.33$ y $\mathrm{SD}=12.21$ y el tercer año básico obtuvo una $\mathrm{M}=64.89$ y $\mathrm{SD}=10.34$. Se evidencia un aumento de la media y un desempeño normal para los tres niveles.

Se puede señalar, además, en relación con todas las pruebas analizadas que aquellas que presentan una mayor complejidad para los niños y niñas son las relacionadas con la adición y sustracción de segundo nivel y los problemas de palabras, identificándose claramente una población de riesgo, como se observa en la tabla 7. Las tareas relacionas con identificación de números, discriminación numérica y número faltante son las que obtienen mejores resultados, inclusive, en alguna de ellas, se puede evidenciar que no existe población en riesgo, ya que todos los niños y niñas alcanzan el puntaje total propuesto para la subtarea. 
Figura 1. Puntaje total prueba EGMA para los cursos de primero a tercero básico

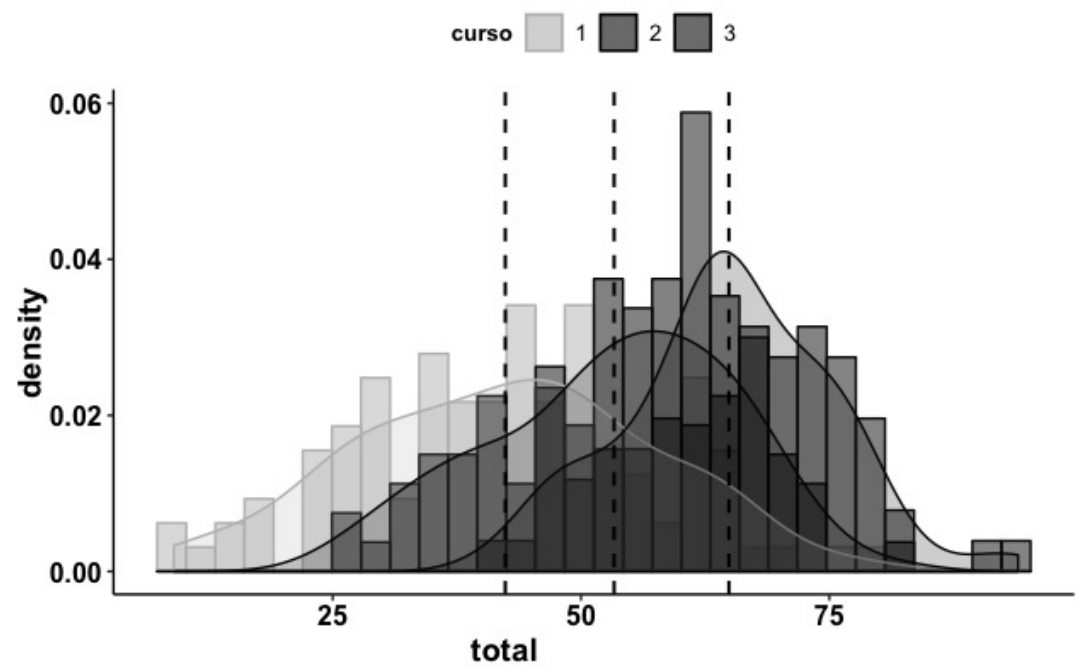

Tabla 7. Estadísticos descriptivos de las tareas EGMA por cursos evaluados

\begin{tabular}{|c|c|c|c|c|c|c|c|c|c|c|}
\hline \multicolumn{3}{|c|}{ Curso } & ID & $\mathrm{DN}$ & $\mathrm{NF}$ & AD N1 & AD N2 & SUS N1 & SUS N2 & PP \\
\hline \multirow[t]{6}{*}{1} & $\mathrm{~N}$ & & 111 & 111 & 111 & 111 & 111 & 111 & 111 & 111 \\
\hline & \multicolumn{2}{|c|}{ Media } & 14,04 & 7,92 & 4,57 & 6,71 & 1,27 & 4,24 &, 57 & 2,68 \\
\hline & \multicolumn{2}{|c|}{ Desviación estándar } & 4,439 & 2,394 & 2,478 & 3,752 & 1,368 & 3,206 & 997 & 1,601 \\
\hline & \multirow[t]{3}{*}{ Percentiles } & 25 & 12 & 7 & 3 & 4 & 0 & 1 & 0 & 2 \\
\hline & & 50 & 15 & 9 & 4 & 7 & 1 & 5 & 0 & 3 \\
\hline & & 75 & 16 & 10 & 6 & 9 & 2 & 7 & 1 & 4 \\
\hline \multirow[t]{6}{*}{2} & $\mathrm{~N}$ & & 91 & 91 & 91 & 91 & 91 & 91 & 91 & 91 \\
\hline & \multicolumn{2}{|l|}{ Media } & 18,26 & 9,25 & 6,32 & 8,13 & 2,12 & 5,46 &, 88 & 2,90 \\
\hline & \multicolumn{2}{|c|}{ Desviación estándar } & 2,649 & 1,179 & 2,467 & 2,952 & 2,265 & 2,945 & 1,237 & 1,660 \\
\hline & \multirow[t]{3}{*}{ Percentiles } & 25 & 18 & 9 & 5 & 6 & 1 & 3 & 0 & 2 \\
\hline & & 50 & 20 & 10 & 7 & 8 & 2 & 6 & 0 & 3 \\
\hline & & 75 & 20 & 10 & 8 & 10 & 3 & 7 & 2 & 4 \\
\hline \multirow[t]{6}{*}{3} & $\mathrm{~N}$ & & 87 & 87 & 87 & 87 & 87 & 87 & 87 & 87 \\
\hline & \multicolumn{2}{|c|}{ Media } & 19,37 & 9,64 & 7,84 & 10,94 & 3,13 & 8,16 & 2,14 & 3,67 \\
\hline & \multicolumn{2}{|c|}{ Desviación estándar } & 1,373 &, 715 & 1,778 & 3,835 & 1,477 & 3,295 & 1,622 & 1,395 \\
\hline & \multirow[t]{3}{*}{ Percentiles } & 25 & 20 & 9 & 7 & 9 & 2 & 6 & 1 & 3 \\
\hline & & 50 & 20 & 10 & 8 & 11 & 3 & 8 & 2 & 4 \\
\hline & & 75 & 20 & 10 & 9 & 13 & 4 & 10 & 3 & 5 \\
\hline
\end{tabular}

Fuente. Elaboración propia.

ID: Identificación numérica, DN: Discriminación Numérica, NF: Número faltante, AD N1: Adición primer nivel, AD N2: Adición segundo nivel, SUS N1: Sustracción primer nivel; SUS N2: Sustracción segundo nivel, PP: problemas de palabra. 
Estudios Pedagógicos XLVI N 1: 301-318, 2020

ESTANDARIZACIÓN DEL INSTRUMENTO EGMA PARA LA EVALUACIÓN DE HABILIDADES NUMÉRICAS INICIALES EN ESTUDIANTES DE EDUCACIÓN BÁSICA DE CHILE

\section{DISCUSIÓN Y CONCLUSIONES}

En el trabajo expuesto se ha presentado el proceso de validación del instrumento EGMA, como herramienta screening, que permita obtener información relevante en los niveles iniciales de la educación básica de cara a la intervención. Con respecto a los valores psicométricos es posible afirmar que hay evidencias en relación con su consistencia interna y análisis factorial que este instrumento reúne las características adecuadas para evaluar las habilidades matemáticas en niños y niñas de primer, segundo y tercer año básico en la población escolar chilena. Estos resultados presentan similares indicadores con respecto a la consistencia interna reportada para una población de 824 estudiantes de segundo a cuarto básico de Nicaragua (Castillo, Castro, Laguna, \& Vijil, 2011), y 4385 estudiantes de segundo y tercero básico de dos países del continente Africano (RTI International, 2014). En ambos estudios, los valores de alpha reportados para las subtareas de número faltante y problemas de palabras registró valores inferiores a .70, lo que se sugiere que los resultados de esta última subtarea sean interpretados considerando el conocimiento y habilidades de los estudiantes del nivel evaluado.

De esta forma se puede mencionar que este instrumento se puede incorporar en el proceso de aprendizaje de los niños y niñas para obtener información significativa sobre los objetivos que están alcanzando los estudiantes de los niveles antes mencionados. En este sentido, se puede ampliar la posibilidad de pruebas que evalúen las habilidades matemáticas, considerando los primeros cursos de la educación básica, ya que a este nivel solo se evidencia la Prueba TEMT-U y TEMA-3, considerando además que la Prueba EGMA tiene un libre acceso y no representa un costo para los centros educativos.

Un elemento importante a considerar es que, en Chile, no existen limitadas evidencias empíricas con respecto al desarrollo de habilidades matemáticas al nivel de primero, segundo y tercer año básico. Una investigación relacionada con la validez y confiabilidad de la prueba TEMT-U, en el año 2012, que concluye que este instrumento puede ser considerado útil para evaluar el desempeño en el área de matemática de estudiantes de segundo ciclo de educación de Párvulos y primer ciclo básico, en la medida que se reportan puntajes comparativos por edad, nivel educativo y dependencia administrativa, además discrimina sobre las competencias matemáticas que presentan mayores dificultad para los niños y niñas de la muestra (Cerda et al., 2012). Con respecto al TEMA-3, que es otro instrumento de evaluación disponible en este nivel, no existen estudios sobre la aplicación en la población chilena, solo a nivel internacional, como en la ciudad de Madrid, donde se pudo identificar la población de tercer grado que manifestaba dificultades concretas en el aprendizaje de las habilidades matemáticas básicas y de esta forma orientar la intervención para su mejora. (Núñez y Gómez, 2011) y en la ciudad de Córdoba, España donde se determinó el nivel de desarrollo del sentido numérico en niños de primer grado de Educación primaria en actividades de matemática informal (Adrián, Jiménez-Fanjul, Maz-Machado, Bracho, García, 2012). Como se muestra en los estudios anteriores, la evaluación temprana es relevante puesto que permite identificar las habilidades y competencias (ver tabla I) que alcanza el alumnado y así dar las respuestas necesarias con información relevante y significativa para adecuar la instrucción de acuerdo con la población que requiere mayor apoyo e intervención.

Las competencias matemáticas que son evaluadas en la prueba EGMA son consistentes con el desarrollo del currículum chileno (MINEDUC, 2012). Así, 
considerando el desempeño de los estudiantes incluidos en la muestra de este estudio en las tareas de identificación numérica, discriminación numérica y número faltante es posible indicar que se presentan buenos resultados. Sin embargo, fue posible identificar una población de estudiantes en riesgo susceptibles a la necesidad de una intervención en las habilidades indicadas. Por ejemplo, para la tarea de identificación numérica se observa que un $27,9 \%$ de estudiantes de primero básico y un $35 \%$ de segundo básico que se desempeña bajo el percentil 25, segmento identificado de riesgo. Lo mismo sucede para la tarea de discriminación numérica donde los estudiantes deberían ser capaces de comparar y ordenar números donde se observa que un $35,1 \%$ del nivel de primero básico y un 38,5\% de segundo año básico conforman la población de riesgo para esta tarea (bajo el percentil 25).

Con respecto a las habilidades de cálculo numérico, el currículum chileno prescribe que a partir de primero básico los estudiantes deben describir y aplicar estrategias de resolución mental para las adición y sustracción y demostrar que comprenden estas operaciones usando un lenguaje cotidiano para describir sus acciones y representándolas de distintas maneras en el ámbito numérico (MINEDUC, 2012). Los resultados en esta investigación indican que los estudiantes se desempeñan bajo lo esperado, donde en la adición de segundo nivel se observa que el 39,6\%, 37,4\% y 34,5\% de los estudiantes observados pueden ser considerados en el grupo de riesgo en los cursos de primer, segundo y tercer año básico respectivamente. Lo mismo sucede con la sustracción de segundo nivel donde la población de riesgo alcanza porcentajes de $66,7 \%, 58,2 \%$ y $36,8 \%$ en los cursos de primer, segundo y tercer año básico.

En este sentido sería importante conocer cuáles son las estrategias que están desarrollando los profesores en el aula, que le permiten a los niños y niñas resolver esta operatoria. Castro (2008), menciona que la acción transformadora en las operaciones constituye un hacer cotidiano haciendo referencia que las operaciones de adición y sustracción se relacionan directamente con el diario vivir, en este sentido es necesario que los niños y niñas puedan tener aprendizajes significativos, que los puedan relacionar con los que diariamente observan y realizan. Otro elemento importante a considerar, tiene relación con las distintas etapas por las que pasan los niños y niñas para adquirir el concepto de operación, según lo expuesto por Cofré y Tapia en el 2003, desde el autor Mialaret, los estudiantes deben pasar por distintos niveles dentro de la operatoria partiendo por la acción real, donde la operación manual debe proceder siempre a la aritmética para terminar traducción simbólica que marca el final del proceso de aprendizaje de las operaciones, ya que el niño utiliza símbolos para traducir las operaciones que ha realizado a nivel concreto. En este sentido, sería interesante confirmar si se está verificando que los niños y niñas van avanzando hacia un nivel de mayor abstracción o se quedan en los niveles concretos, donde necesitan ayuda de elementos para poder resolver. Esto se relaciona directamente con la práctica docente que según Flores (2005) se vincula más a una concepción tradicional de enseñanza. Las concepciones y enseñanzas de los docentes sobre suma y resta, y los contenidos específicos de matemáticas, determina en gran medida su actuar dentro y fuera del aula, sus trabajos estarán basados a partir de sus propios conocimientos formales e informales y vida cotidiana (Pérez y Vera, 2012).

Con respecto a las habilidades para resolución de problemas de palabras, se puede observar los estudiantes presentan dificultades para ejecutar este tipo de ejercicios evidenciando que el $48,6 \%, 46,2 \%$ y $43,7 \%$ de los estudiantes evaluados se encuentra dentro 
del grupo de riesgo en los cursos de primer, segundo y tercer año básico respectivamente. El diseño de los ejercicios de problemas de palabras consideradas en la prueba EGMA están relacionadas con la vida cotidiana y con números del ámbito entre uno y diez, obedeciendo a distintos tipos y niveles de problemas, relacionados con lo que establece el currículum de Chile. Los resultados pueden evidenciar que no existe consistencia con respecto a la instrucción de esta habilidad de forma transversal dentro de la asignatura de matemática como lo establece los planes y programas del año 2012, en los cuales queda definido como una habilidad que se debe integrar con los objetivos de aprendizaje, o queda limitada a resolver problemas al finalizar el trabajo con las distintas operaciones.

La disponibilidad de herramientas de evaluación adecuadas presenta una gran relevancia puesto que permite conocer las trayectorias en el proceso de aprendizaje y establecer de esta forma, medidas de acción que permitan ir en ayuda principalmente de aquellos estudiantes que están presentando mayores dificultades (Baroody, 1997). En este sentido, se ha reportado que los resultados generados utilizando EGMA son valiosos para la toma de decisiones a nivel del diseño de políticas educativas, informar el impacto de reformas curriculares y el diseño de programas de intervención (Platas, Ketterlin-Geller, \& Sitabkhan, 2016). Particularmente en el ámbito del desempeño docente, la adecuada detección temprana de dificultades académicas de los estudiantes permite proyectar un trabajo más ordenado y sistemático en el proceso de aprendizaje de los niños y niñas. De esta forma sería interesante y necesario contar con una herramienta que entregue este tipo de información a los docentes pudiendo optar así a la aplicación de una evaluación temprana a los estudiantes y establecer claramente los distintos niveles de aprendizaje y más que eso, ya que la mirada no está dirigida en clasificar a un grupo de niños, sino más bien a poner el énfasis en las ayudas que se brindan para que ellos puedan superar las dificultades y no vayan quedando con vacíos, que por la actual dinámica escolar, muchas veces van pasando curso tras curso, sin detectar a tiempo las necesidades que están presentando.

Los resultados reportados en esta investigación demuestran la validez y pertinencia de la prueba EGMA para ser utilizada como una herramienta de screening tipo CBM para la población de estudiantes de Chile. En este sentido, cabe mencionar que como limitantes de este trabajo se observa una carencia de evidencia empírica relacionada con la evaluación de las habilidades matemáticas tempranas de los primeros niveles de enseñanza básica, que permita lograr un análisis comparado con respecto al desempeño de estudiantes en los mismos niveles evaluados en este estudio. La muestra informada en esta investigación es la primera prospección de la prueba EGMA aplicada a la población de estudiantes de Chile, es deseable que futuros estudios se logre desarrollar una investigación con una mayor cantidad de estudiantes que represente adecuadamente la diversidad de centros educativos y de diferentes regiones del país.

\section{AGRADECIMIENTOS Y FINANCIAMIENTO}

Los autores agradecen a los docentes, administradores y estudiantes de los centros educativos participantes los que con su generosa cooperación hicieron este estudio posible. La investigación fue parcialmente financiada por el proyecto CONICYT, FONDECYT REGULAR 1161213 del Ministerio de Educación de Chile y el Ministerio de Economía y Competitividad de España, Programa Ramón y Cajal, RYC-2014-16948. 


\section{REFERENCIAS BIBLIOGRÁFICAS}

Adrián, C., Jiménez-Fanjul, N., Maz-Machado, A., Bracho, R. y García, T. (2012). Matemática informal y sentido numérico en escolares de primer ciclo de E. Primaria. Recuperado de http:// thales.cica.es/xivceam/actas/pdf/com09.pdf

Agencia de Calidad de la Educación (2015). Informe Nacional de Resultados TERCE. Recuperado de http://archivos.agenciaeducacion.cl/TERCE_informefinal.pdf

Agencia de Calidad de la Educación (2015). Resultados TIMMS Chile. Recuperado de http:// archivos.agenciaeducacion.cl/TIMMS_presentacion_BAJA.pdf

Agencia de Calidad de la Educación (2017). Resultados educativos 2017. Santiago, Chile. Recuperado de http://archivos.agenciaeducacion.cl/PPT_Conferencia_ER_2017_web_3.pdf

Agencia de Calidad de la Educación (2018). Evaluaciones nacionales e internacionales de aprendizaje. Recuperado de http://archivos.agenciaeducacion.cl/Panorama_Evaluaciones_nacionales_e_ internacionales_V03_01MAR.pdf

Agencia de Calidad de la Educación (2018). Resultados educativos 2017. Recuperado de http:// archivos.agenciaeducacion.cl/PPT_Conferencia_ER_2017_web_3.pdf

Andrews, P. \& Sayers, J. (2013). Foundational Number Sense : A Framework For Analysing Early Number - Related Teaching (pp. 17-26).

Aunio, P. \& Niemivirta, M. (2010). Predicting children's mathematical performance in grade one by early numeracy. Learning and Individual Differences, 20(5), 427-435. http://doi.org/10.1016/j. lindif.2010.06.003

Baroody, A. (1997). El pensamiento matemático de los niños. VisorsDis. Madrid, España.

Castillo, M., Castro, V., Laguna, J. R. \& Vijil, J. (2011). Informe de resultados: EGMA Nicaragua. RTI Internacional.

Castro, E. (2008). Didáctica de la Matemática e la Educación Primaria. Editorial Síntesis, S.A. Madrid, España

Cerda, G., Pérez, C., Moreno, C., Núñez, K., Quezada, E., Rebolledo J. y Sáez, S. (2012). Adaptación española del Test de Evaluación Matemática temprana de Utrecht en Chile. Estudios Pedagógicos XXXVIII(1), 235-253. Recuperado de http://www.scielo.cl/pdf/estped/v38n1/art14.pdf

CIASES Y RTI Internacional. (2011). Informe resultados EGMA: Nicaragua. Recuperado de https:// ierc-publicfiles.s3.amazonaws.com/public/resources/Nicaragua\%20EGMA\%20report.pdf

Clarke, B., \& Shinn, M. R. (2004). A preliminary investigation into the identification and development of early mathematics curriculum-based measurement. School Psychology Review, 33(2), 234248. http://doi.org/Article

Clarke, B., Baker, S., Smolkowski, K. \& Chard, D. J. (2008). An analysis of early numeracy curriculum-based measurement: Examining the role of growth in student outcomes. Remedial and Special Education, 29(1), 46-57. http://doi.org/10.1177/0741932507309694

Clements, D. H., \& Sarama, J. (2009). Learning and Teaching Early Math: The Learning Trajectories Approach. New York: Routledge.

Cofré, A., Tapia, A. (2003). Cómo desarrollar el razonamiento lógico matemático. Fundación Educacional Arauco. Editorial Universitaria. Santiago de Chile.

Deno, S. L. (2003). Curriculum-based measures: Development and perspectives. Assessment for Effective Intervention, 28(4), 3-12. https://doi.org/10.1177/073724770302800302

Field, A. (2013). Discovering Statistics Using SPSS. SAGE Publications (4th ed.). London: Sage Publications. http://doi.org/10.1111/j.1365-2648.2007.04270_1.x

Flores, A. (2005). ¿Cómo saben los alumnos que lo que aprenden en matemáticas es cierto?: Un estudio exploratorio. Educación Matemática, 17(3), 5-24. Recuperado de https://www.redalyc. org/comocitar.oa?id=40517302

Geary, D. C. (2011). Cognitive predictors of achievement growth in mathematics: A 5-year longitudinal study. Developmental Psychology, 47(6), 1539-1552. http://doi.org/10.1037/a0025510 
Estudios Pedagógicos XLVI N 1: 301-318, 2020

ESTANDARIZACIÓN DEL INSTRUMENTO EGMA PARA LA EVALUACIÓN DE HABILIDADES NUMÉRICAS INICIALES EN ESTUDIANTES DE EDUCACIÓN BÁSICA DE CHILE

Göbel, S. M., Watson, S. E., Lervåg, A. \& Hulme, C. (2014). Children's Arithmetic Development: It Is Number Knowledge, Not the Approximate Number Sense, That Counts. Psychological Science, 25(3), 789-798. http://doi.org/10.1177/0956797613516471

Hernández, J. (2019). ULLRtoolbox. https://sites.google.com/site/ullrtoolbox/

Jacobi-Vessels, J. L., Todd Brown, E., Molfese, V. J. \& Do, A. (2016). Teaching Preschoolers to Count: Effective Strategies for Achieving Early Mathematics Milestones. Early Childhood Education Journal, 44(1), 1-9. http://doi.org/10.1007/s10643-014-0671-4

Jordan, N. C., Glutting, J., Ramineni, C. \& Watkins, M. W. (2010). Validating a number sense screening tool for use in kindergarten and first grade: Prediction of mathematics proficiency in third grade. School Psychology Review, 39(2), 181-195.

León, S. C., Jiménez, J. E., García, E., Gutiérrez, N., \& Gil, V. (2020). Universal Screening in Mathematics for Spanish Students in First Grade. Learning Disability Quarterly. doi: $10.1177 / 0731948720903273$

MINEDUC. (2012). Bases Curriculares. Matemática. Unidad de Currículum y evaluación, Ministerio de educación. Santiago de Chile.

MINEDUC. (2014). Estándares Indicativos de desempeño para los establecimientos Educacionales $\mathrm{y}$ sus sostenedores. Recuperado de http://archivos.agenciaeducacion.cl/documentos-web/ Estandares_Indicativos_de_Desempeno.pdf

MINEDUC. (2016). Plan de aseguramiento de la calidad escolar 2016-2019. Recuperado de http:// portales.mineduc.cl/plandeaseguramiento/files/assets/common/downloads/Plan\%20de\%20 Aseguramiento.pdf

Navarro, J., Aguilar, M., Marchena, E., Alcalde, C. y García, J. (2010). Evaluación del conocimiento temprano en una muestra de $3^{\circ}$ de Educación infantil. Recuperado de http://www. revistaeducacion.educacion.es/re352/re352_27.pdf

Núñez, M., Gómez, P. (2011). Habilidades matemáticas básicas en alumnos de $3^{\circ}$ de infantil. Detección temprana de dificultades de aprendizaje y orientaciones para la intervención. Revista Diálogo Educacional, 11(32),83-105. Recuperado de https://www.redalyc.org/pdf/1891/189118887006.pdf

Platas, L. M., Ketterlin-Geller, L. R. \& Sitabkhan, Y. (2016). Using an Assessment of Early Mathematical Knowledge and Skills to Inform Policy and Practice: Examples from the Early Grade Mathematics Assessment. International Journal of Education in Mathematics, Science and Technology, 4(3), 163-173. http://doi.org/10.18404/ijemst.20881

Pérez, G., Vera, J. (2012). Lógica subyacente de la enseñanza de la suma y resta en profesores de primero a tercer grado escolar. Revista Tiempo de educar, 13(25), 51-81. Recuperado de https:// www.redalyc.org/pdf/311/31124808003.pdf.

Purpura, D. J., \& Lonigan, C. J. (2015). Early Numeracy Assessment: The Development of the Preschool Numeracy Scales. Early Education and Development, 26(2), 286-313. http://doi.org /10.1080/10409289.2015.991084

RTI International. (2014). Early Grade Mathematics Assessment (EGMA) Toolkit.

UNESCO (2016). Informe de resultados TERCE. Logro de aprendizaje. Recuperado de http:// unesdoc.unesco.org/images/0024/002435/243532S.pdf

Vanbinst, K., Ansari, D., Ghesquière, P. \& Smedt, B. De. (2016). Symbolic numerical magnitude proceing is as important to arithmetic as phonological awarene is to reading. PLOS ONE, 11(3), 1-11. http://doi.org/10.1371/journal.pone.0151045 